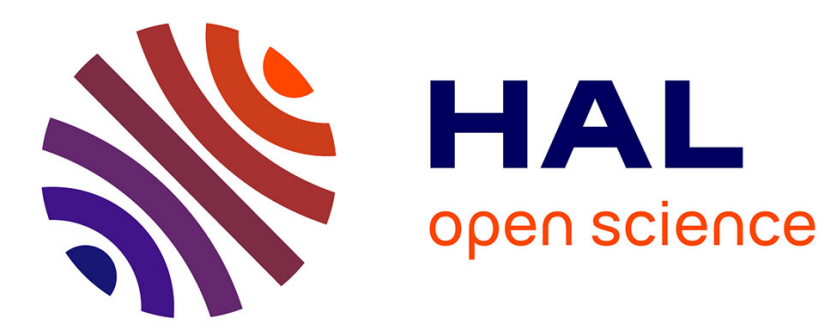

\title{
DIFFUSION PROCESSES IN A LIQUID MIXTURE OF LITHIUM ISOTOPES
}

\author{
Marco Omini
}

\section{To cite this version:}

Marco Omini. DIFFUSION PROCESSES IN A LIQUID MIXTURE OF LITHIUM ISOTOPES.

Philosophical Magazine, 2009, 89 (01), pp.1-25. 10.1080/14786430802422006 . hal-00513962

\section{HAL Id: hal-00513962 \\ https://hal.science/hal-00513962}

Submitted on 1 Sep 2010

HAL is a multi-disciplinary open access archive for the deposit and dissemination of scientific research documents, whether they are published or not. The documents may come from teaching and research institutions in France or abroad, or from public or private research centers.
L'archive ouverte pluridisciplinaire HAL, est destinée au dépôt et à la diffusion de documents scientifiques de niveau recherche, publiés ou non, émanant des établissements d'enseignement et de recherche français ou étrangers, des laboratoires publics ou privés. 


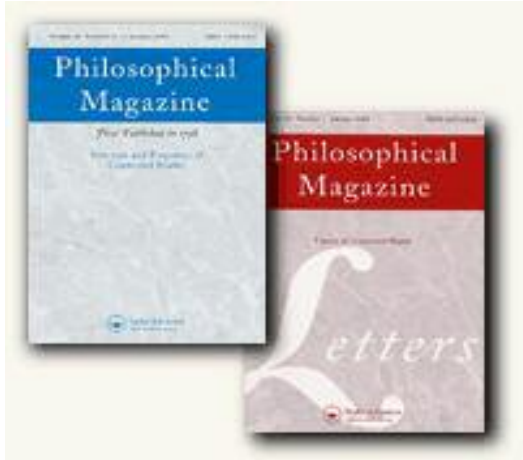

DIFFUSION PROCESSES IN A LIQUID MIXTURE OF LITHIUM ISOTOPES

\begin{tabular}{|r|l|}
\hline Journal: & Philosophical Magazine \& Philosophical Magazine Letters \\
\hline Manuscript ID: & TPHM-08-Jun-0219 \\
\hline Journal Selection: & Philosophical Magazine \\
\hline Date Submitted by the \\
Author: & 10-Jun-2008 \\
\hline Complete List of Authors: & Omini, Marco; Politecnico of Torino, Physics \\
\hline Keywords: & diffusion, liquid metals \\
\hline Keywords (user supplied): & isotope effects \\
\hline &
\end{tabular}

\section{s) ScholarONE \\ Manuscript Central}




\title{
DIFFUSION PROCESSES IN A LIQUID MIXTURE OF LITHIUM ISOTOPES
}

\author{
M. Omini \\ Dipartimento di Fisica and CNISM, Politecnico di Torino \\ Email: marco.omini@polito.it
}

\begin{abstract}
.
The quantum mechanical theory of diffusion, as recently developed for liquid metals [M. Omini, Phil. Mag. 86, 1643 (2006) and 87, 5249 (2007)], is now presented in a final version, which improves and clarifies the original formulation. The new formulation uses a convergent variational method to solve the transport equation and confirms, through a rigorous analysis, the validity of the approximations which were introduced in the quoted papers. As a consequence, all the previous calculations, concerning the self diffusion coefficients of liquid lithium isotopes, are automatically confirmed. The possibility of obtaining, as a limit of a convergent procedure, the true solution of the transport equation allows a reliable prediction of all the isotope effects. In particular, it provides the numerical values of the interdiffusion coefficients of ${ }^{6} \mathrm{Li}$ and ${ }^{7} \mathrm{Li}$, which were not explicitly deduced in the previous works. The full consistency of these values with those measured by Feinauer et al. [A. Feinauer, G. Majer and A. Seeger, J. Phys. Condensed Matter, 6 L355 (1994)] represents a further support to the theory. The conclusion is that the whole set of experimental data on liquid lithium, as provided by the above authors, finds its theoretical explanation within the experimental uncertainties.
\end{abstract}




\section{1- Introduction.}

In the theory of diffusion developed earlier for liquid metals [1,2] a basic role was assigned to collective oscillations, which, in fact, were invoked to explain the observed behaviour of liquid lithium. The weight of collective modes was found to be relevant in determining the temperature dependence of the self-diffusion coefficients of ${ }^{6} \mathrm{Li}$ and ${ }^{7} \mathrm{Li}$ and of the corresponding isotope effect. While this effect was widely discussed in the quoted papers, the problem presented by the interdiffusion coefficients of the two isotopes was only marginally touched. It was only pointed out that, as a consequence of the above theory, the ratio between the coefficients of ${ }^{6} \mathrm{Li}$ diffusing through pure ${ }^{7} \mathrm{Li}$ and of ${ }^{7} \mathrm{Li}$ diffusing through pure ${ }^{6} \mathrm{Li}$ should be about unity, and that the experimental data of Feinauer, Majer and Seeger $[3,4]$ were in agreement with this prediction. Such a result, however, has not to be considered as a decisive element in favour of the same theory. In fact, the classical extension of the Enskog formula predicts the dependence of the diffusivity on the reduced mass of the two isotopes [5] and, therefore, the identity of the two interdiffusion coefficients: a result of this kind, corresponding to a ratio rigorously equal to unity, would similarly be, within the experimental uncertainties, in agreement with experiment. A significant proof would be represented by a test of the absolute values of the two coefficients, but no attempt was made to deduce these values from first principles. A theoretical effort in this direction is precisely the aim of the present paper.

As an introduction to the following discussion, it is necessary to point out that, on the one hand, the isotope effects are small and, on the other hand, all the expressions previously given for the diffusion coefficients [1,2] derive from an approximate solution of the transport equation. In order to test the theoretical predictions through a comparison with the experimental data, it is imperative to eliminate from these predictions any unwanted error due to the mathematical difficulty of solving the above equation.

The method adopted earlier to solve the transport equation was based on a variational procedure, applied to a trial function with some adjustable parameters by which the approximate solution was represented. The trial function was chosen as the simplest function satisfying the asymptotic behaviours for $q \rightarrow 0$ and $q \rightarrow \infty$ (where $q$ is the wavevector of the diffusing particle). Such a choice does not necessarily imply a good representation of the solution in the intermediate range about $q_{t h}$ (the value of $q$ corresponding to the mean thermal energy), which is precisely the range one expects to bring the main contribution to the diffusivity. Under these conditions it is not possible to know the level of reliability characterizing the final results of the theory. They can be trusted only if we show that a diffusion coefficient is weakly depending on the particular form of the trial function and, therefore, weakly affected by deviations of this function from the true (unknown) solution of the transport equation.

Owing to the numerical calculations performed in the mentioned papers, one is led to believe that things go precisely in this way: the consistency of the results obtained through the use of two different trial functions, as employed when the isotope effect of self-diffusion was investigated, represents a strong argument in favour of the above conclusion. However, a rigorous proof has not been given and seems to be not only advisable, but necessary if the effects we want to discuss are small: this is precisely the case presented by a theory where one wishes to make a distinction between the 
interdiffusion coefficients of ${ }^{6} \mathrm{Li}$ and ${ }^{7} \mathrm{Li}$, which are very close. The proof will be given after a critical revision of all the assumptions on which the quantum mechanical theory of diffusion is founded. In fact, it is convenient to recall and reconsider the basic features of the theory, in order to throw new light on some points which were not sufficiently clarified in the previous papers. The corresponding discussion is contained in sects 2 and 3, which provide a full justification of the results obtained in the original treatment and, at the same time, represent a final setting of the theoretical framework from which these results can be derived.

\section{2- Collective oscillations in thermal equilibrium.}

The starting point is a liquid of $N$ atoms, where $3 N^{\prime}$ modes of collective harmonic oscillation are in thermal equilibrium. Each mode is characterized by a wavevector $\boldsymbol{k}$ and frequency $\omega=s k$, where $s$ is the sound velocity. There is a maximum frequency $\omega_{m}$, corresponding to the mode with the highest value of $k$. The modes are distributed over the frequency range between 0 and $\omega_{m}$, according to a spectral density $\mathcal{N}_{m}(\omega)$, defined in such a way that $\mathcal{N}_{m}(\omega) \mathrm{d} \omega$ represents the number of modes in $\mathrm{d} \omega$. Since each mode corresponds to a degree of freedom, there is a total number $3 N^{\prime}$ of degrees of freedom involved in harmonic motions and $\mathcal{N}_{m}(\omega) \mathrm{d} \omega$ of these vibrational degrees are associated to the interval $\mathrm{d} \omega$. All the remaining degrees refer to noncollective motions of single particles.

As a result of the interference of all the modes in thermal equilibrium, we can only expect a system of oscillating atoms, characterized by the following properties:

a) the total momentum of the system is zero, because for each mode with wavevector $\boldsymbol{k}$ a corresponding mode with wavevector $-\boldsymbol{k}$ is present in the liquid;

$b)$ the system has $3 N^{\prime}$ vibrational degrees, distributed over the frequency range between 0 and $\omega_{m}$ according to the spectral density $\mathcal{N}_{m}(\omega)$.

Now, these are simply the properties of a system of $N^{\prime}$ atoms, vibrating around fixed equilibrium positions, and distributed over the above frequency range with a spectral density $\mathcal{N}(\omega)=\mathcal{N}_{m}(\omega) / 3$. In fact, since each of these atoms brings three degrees of freedom, the number of vibrational degrees associated to the interval $\mathrm{d} \omega$ is precisely $3 \mathcal{N}(\omega) \mathrm{d} \omega=\mathcal{N}_{m}(\omega) \mathrm{d} \omega$, as expected. This suggests a picture where $N^{\prime}$ atoms of the liquid behave as three-dimensional oscillators, while the remaining $N^{\prime \prime}=$ $N-N^{\prime}$ atoms perform the (translational) motions associated to the non-vibrational degrees. The $N^{\prime \prime}$ atoms undergo mutual scattering processes and can be considered as free particles in the time interval between two successive collisions. We will assume these atoms to be distinguishable, in agreement with the use of Boltzmann statistics, by which their distribution will be described. For self-consistency of the theory, this requires all the $N$ atoms to be distinguishable particles.

The choice of the $N^{\prime}$ atoms is not unique. The number of possible choices is represented by the number of ways, $W$, by which one can form a set of $N^{\prime}$ atoms, starting from a total number of $N$ distinguishable atoms. Each choice corresponds to a quantum state of the system, where only the atoms of the selected set behave as oscillation centres, that is become solid-like atoms, while the remaining atoms perform unbounded motions in the whole liquid volume (liquid-like atoms).

The solid-like atoms perform harmonic oscillations around equilibrium positions that we assume to be randomly spread over the liquid volume (an assumption following 
from the fact that $N^{\prime}$ is presumed to be small, so that we exclude mutual correlations between oscillators). These atoms are reminiscent of the oscillators originally introduced by Mott [6] to explain the electrical conductivity of liquid metals. However, while in Mott' $\mathrm{s}$ theory all the atoms were assimilated to harmonic oscillators, here only a fraction $x=N^{\prime} / N$ of atoms can be treated in this way.

Apparently, the assumption of $N^{\prime}$ Mott oscillators seems to contradict the intuitive idea that for all the atoms of the liquid the motion should be a combination of oscillatory motion and random translational motion, when collective modes are present. Actually, the contradiction does not exist and the reason is typically quantum mechanical. Each of the $W$ states represents a pure quantum state, in the sense that it refers to $N^{\prime}$ atoms performing pure oscillations and $N^{\prime \prime}$ atoms performing pure translational motions. Since none of the $W$ sets of $N^{\prime}$ atoms is privileged with respect to the others, all the $W$ states must be equivalent and, therefore, belong to the same energy. It follows that any linear combination of these states is still a quantum state with the same energy. All the coefficients of the combination must be equal because all the $W$ states must be treated in the same way. As a consequence, the quantum state of the real liquid is a mixture of these states and the liquid can be found with the same probability in any of them. The conclusion is that we cannot specify the set of $N^{\prime}$ atoms which behave as Mott oscillators. In other words, we cannot know whether a selected atom $a$ is a solid-like atom or not. Since, among the $W$ sets, there are $W N^{\prime} / N$ sets containing atom $a$, we can only say that this atom has a probability $N^{\prime} / N$ of being a solid-like atom and a probability $1-\left(N^{\prime} / N\right)$ of being a liquid-like atom. In this way no atom is characterized by a pure oscillatory motion and no atom by a pure translational motion; both the dynamical behaviours are simultaneously taken into account, though with different weights, in such a probabilistic description of the atomic motion. This is in full agreement with our expectations.

In a given pure state, each of the $N^{\prime}$ atoms oscillates with a frequency which varies stochastically with time over the range between 0 and $\omega_{m}$, but in such a way that, at any time, the total number of atoms oscillating with frequency in $\mathrm{d} \omega$ is $\mathcal{N}(\omega) \mathrm{d} \omega$. All the $N^{\prime}$ atoms are equivalent, because the stochastic behaviour is the same for all of them. As a consequence, if any two of these atoms ( $a$ and $b$ ) are interchanged, the two dynamical behaviours corresponding to the system before and after the interchange are undistinguishable. All the ways leading to a set of $N^{\prime}$ oscillating atoms, and deriving from mutual interchanges of the atoms belonging to the set, are undistinguishable. This means that no configurational entropy term must be associated to the possibility of performing such interchanges. In other words, the $N^{\prime}$ atoms behave as if they were undistinguishable particles, precisely as assumed earlier [2].

Things go differently if the interchange involves two atoms, $a$ and $b$, characterized by different dynamical behaviours. This is the case when $a$ is a solid-like atom and $b$ a liquid-like atom. Since the atoms $a$ and $b$ are assumed to be distinguishable particles, the two dynamical behaviours corresponding to the system before and after the interchange are distinguishable. Let us point out that the above case corresponds to the transition from a particular set of $N^{\prime}$ atoms (including $a$ and excluding $b$ ) to another particular set (including $b$ and excluding $a$ ). In this way we have shown that different sets correspond to distinguishable ways of realizing a distribution of $N^{\prime}$ 
oscillating atoms. It follows that the $W$ sets correspond to a configurational entropy $S=k_{\mathrm{B}} \ln W$.

To determine the value of $N^{\prime}$ at equilibrium, we have to write the total free energy of the system as a function of this variable. To this purpose, it is convenient to regard the atomic motions as responsible for the difference between the instantaneous structure of the system (which changes stochastically with time) and the structure of the supercooled liquid at $T=0$, corresponding to the absence of any motion. In this way it is possible to split the total Hamiltonian into a dynamical part and a static part, the latter being referred to the supercooled liquid. Correspondingly, the free energy will contain a static contribution $F_{s}$ and a dynamical contribution $F_{d}$. These must be added to the configurational contribution $F_{\text {conf }}=-k_{\mathrm{B}} T \ln W$, which accounts for the $W$ choices we have at disposal to define the $N^{\prime}$ atoms.

A first term contributing to $F_{d}$ is the vibrational free energy $F_{v i b}$. To express this term, we must specify $\mathcal{N}(\omega)$ and $\omega_{m}$ as a function of $N^{\prime}$. If the wavevectors of the collective modes are stuffed with the highest possible density inside a sphere of the reciprocal space with radius $K_{m}$ (an assumption justifiable through a principle of minimum free energy [2]), one finds $\omega_{m}=s K_{m}$ and $\mathcal{N}(\omega)=3 N^{\prime} \omega_{m}{ }^{-3} \omega^{2}$, where

$$
K_{m}=\left(18 \pi^{2} v^{-1} N^{\prime} / N\right)^{1 / 3}
$$

$v$ being the atomic volume. At this point it would be easy to write the free energy of all the harmonic oscillators represented by the solid-like atoms: however, such a free energy would not provide the whole contribution to $F_{v i b}$. One must add, in fact, the contribution of the oscillating field that each of the above atoms engendres in its neighbourhood, that is in the surrounding system of liquid-like atoms. This contribution accounts for the dynamical correlations between the two kinds of atoms and can be simply described by assuming that a liquid-like atom, under the effect of the field, acquires a probability $p$ of becoming itself a Mott oscillator. Thus, on the average, each solid-like atom is surrounded by $\xi=v p$ Mott oscillators, $v$ being the number of nearest neighbours. The origin of these oscillators has to be sought in the scattering processes which allow the propagation, through the system of liquid-like atoms, of the oscillating field created by a solid-like atom (a system of free particles, not subject to scattering processes, could not propagate a disturbance). The parameter $p$ accounts for a probabilistic description of this field, more precisely for the modification produced in the scattering regime by the creation of a solid-like atom. The $\xi$ Mott oscillators are simply an approximate but useful tool to write the corresponding modification of the total free energy. In our simple model this appears as a vibrational free energy term, and is automatically taken into account by $F_{v i b}$ if each solid-like atom is associated to $1+\xi$ oscillators of the same frequency. In principle, $\xi$ depends on the oscillation frequency, but only the value corresponding to the highest frequency $\omega_{m}$ is actually required [2].

It would be difficult to provide an explicit expression for $p$ in terms of microscopic properties of the liquid. However, the temperature dependence of this parameter can be qualitatively predicted by making reference to a fluid described by a classical continuum. For such a fluid the basic quantity determining the coupling between an oscillating point source and its environment is represented by the viscosity $\eta$ : a high 
value of $\eta$ implies a short range oscillating field in the neighbourhood of the source and then a weak coupling, and viceversa. Thus $p$ is expected to be a decreasing function of $\eta$ : since $\eta$ in liquids decreases with $T$, it follows that $\xi=v p$ must rise with temperature. The same result can be deduced from the temperature dependence of the probability rates for scattering processes (see sect. 3). Nevertheless, we insist on the above simple description in terms of viscosity because it provides a further support to the consistency of the whole theory. In fact, in Appendix A we show, through dimensional arguments, that the equilibrium value of the ratio $N^{\prime} / N$, as obtainable in terms of $\eta$ for the continuum model, is independent of the atomic mass, so that the same property holds for $K_{m}$ (see equation (1)). This is in full agreement with our expectations, since $K_{m}$ plays the role of a Debye radius and must depend only on the liquid structure.

A second contribution to $F_{d}$, here denoted by $F_{t r}$, derives from all the particles which perform random translational motions. The number of these particles is obtained by subtracting from $N$ the total number of oscillators accounted for by $F_{v i b}$. The particles are assumed to move in a background constant potential and undergo mutual collisions by which they are maintained in thermal equilibrium. For a gas of particles of this kind, the free energy is easily deduced from standard books of statistical Thermodynamics. Details of the calculation leading to $F_{t r}$ are found in the quoted reference.

To arrive at $N^{\prime}$, one has simply to minimize with respect to this variable the total free energy $F=F_{\text {conf }}+F_{v i b}+F_{t r}+F_{s}$, where $F_{s}$ accounts for the energy change $\delta U_{s}$ and the entropy change $\delta S_{s}$, accompanying in the supercooled liquid the structure change connected with the transition from a liquid-like to a solid-like atom (strictly, one should minimize the Gibbs free energy, but this would simply add to $\delta U_{s}$ a term $p \delta v$, accounting for the volume change $\delta v$ produced by the same transition, and completely negligible at room pressure). An expression in terms of $\delta U_{s}$ and $\delta S_{s}$ was, in fact, explicitly obtained for the equilibrium value of $N^{\prime} / N$ [2]. Notwithstanding the difficulty connected with the determination of $\delta U_{s}$ and $\delta S_{s}$, it was possible to use this expression to predict an upper limit for $N^{\prime} / N$ at the melting point and show that, for lithium, this limit is about $5 \cdot 10^{-2}$. From the same expression one also deduces that $N^{\prime} / N$ decreases strongly with $\xi$. Since $\xi$ increases with $T$, it follows that a temperature rise is expected to produce a strong diminution of $N^{\prime} / N$. This result is in full agreement with the corresponding diminution of $K_{m}$, as deduced earlier from our interpretation of diffusivity data [1,2] .

\section{3- Pseudopotential and structure.}

The model of sect.2 is useful to discuss the equilibrium value of $N^{\prime}$ and to show the existence of an upper limit for this value. The model is not directly involved, however, in the theory of diffusion, where $N^{\prime}$ appears as an adjustable parameter, to be determined by a comparison between theoretical and experimental diffusivities. It will be a task of the theory to show that the value of $N^{\prime}$ determined in this way is fully consistent with the above upper limit.

In order to discuss the scattering processes responsible for the diffusion of solute atoms, one needs a theory based on an explicit form of the interatomic pair potential $\Phi(r)$. This can be split into a short range part $V_{\mathrm{hc}}(r)$, describing the hard core 
interaction between atoms, and a soft potential $V_{\mathrm{s}}(r)$, extended over a range higher than the mean interatomic distance, and responsible for elastic waves.

Let us start from a system of atoms where only the short range potential is active. In this case the scattering processes due to $V_{\mathrm{hc}}(r)$ are responsible for the propagation of any dynamical disturbance through the system. The coupling between the disturbance and the environment will be called a dynamical correlation, of which an example is represented by the coupling between a solid-like atom and the surrounding liquid-like atoms. For any atom, the probability of undergoing a scattering process increases with $T$, so that the strength of the coupling, as measured by $\xi$ in the above example, is expected to rise with temperature.

Let us now switch on the soft interaction $V_{\mathrm{s}}(r)$ between an atom $a$ and an atom $b$ belonging to the system. Since $V_{\mathrm{s}}(r)$ is a long range potential, the scattering process to which such interaction gives rise can be accomplished only if the trajectories of $a$ and $b$ extend over a distance higher than the interatomic distance. However, the required distance is much higher than the mean free path due to $V_{\mathrm{hc}}(r)$ : this implies a high probability, both for $a$ and $b$, of undergoing a short range collision before accomplishing the corresponding trajectory, in which case the scattering process due to $V_{\mathrm{s}}(r)$ is hindered. The probability rises with $T$, so that at high temperatures there is a drastic reduction of the number of soft potential scattering processes which can be fully accomplished. As a consequence, the contribution of the soft potential to the scattering processes which determine the diffusivity is expected to decrease with temperature, while that of the short range potential is expected to rise.

Both the above conditions are automatically fulfilled if $V_{\mathrm{s}}(r)$ is assumed to be represented by an effective temperature dependent potential $V(r)$. This is defined in such a way that all the scattering processes to which it gives rise are fully accomplished; however, $V(r)$ by definition is assumed to decrease strongly with $T$, so that the weight of these processes is correspondingly reduced. More detailed information on $V(r)$ can be obtained from the analysis of collective oscillations, because the existence of elastic waves is just due to the soft part of the pair potential. To this purpose we use the original Percus and Yevick formulation [7], according to which each Fourier component of the effective potential is linked to a corresponding mode of collective oscillation. In this way the existence of $3 N^{\prime}$ modes in thermal equilibrium becomes an important source of information on the form of $V(r)$.

A liquid where $3 N^{\prime}$ degrees of freedom are associated to collective modes of oscillation (in thermal equilibrium with the translational motions related to the remaining degrees) can be described as a system, with Hamiltonian $H^{0}$, where the atoms are embedded in a uniform external potential $-u^{0}$ and interact through a pair potential $V(r)$ characterized by $3 N^{\prime}$ Fourier components. Such a result follows precisely from the Percus and Yevick formulation, when applied to a pair potential of this kind. In fact, the corresponding form of $H^{0}$ is found to contain, in addition to purely kinetic terms, the sum of $3 N^{\prime}$ Hamiltonians of harmonic oscillators [2]: as shown by equation (7) of the quoted reference, these can be precisely interpreted as the Hamiltonians of the $3 N^{\prime}$ collective modes defined in sect. 2, if the $3 N^{\prime}$ wavevectors involved in the Fourier expansion of $V(r)$ are identified with the wavevectors contained in the sphere of radius $K_{m}$. Through the background potential $-u^{0}$ one accounts for the presence of a structure, for which the energy of the liquid is lower than the energy of a disordered system (see Appendix B). 
If $V(r)$ has Fourier coefficients $V_{k}$ (see equation (B1) for their exact definition) the $3 N^{\prime}$ oscillators appearing in $H^{0}$ have frequencies $\omega_{k}=s k$, where $s=\left(k_{B} T / m a_{k}\right)^{1 / 2}$ and the structure factor $a_{k}$ is linked to $V_{k}$ by the Ascarelli, Harrison and Paskin relation [8]. In the limit $k \rightarrow 0$, which is of interest for the narrow interval $0 \leq k \leq$ $K_{m}$ to which the collective modes are confined, such a relation is

$$
V_{0}=v k_{\mathrm{B}} T\left(a_{0}^{-1}-1\right)
$$

and all the coefficients $V_{k}$ can be confused with $V_{0}$. Thus, if $V_{0}$ is evaluated by inserting into equation (2) the experimental value of $a_{0}$ and $V(r)$ is defined by Fourier coefficients $V_{k}=V_{0}$ for $k \leq K_{m}$ and $V_{k}=0$ for $k>K_{m}$, the Hamiltonian $H^{0}$ describes a condensed system containing the required collective modes, characterized by the isothermal sound velocity $s=\left(k_{B} T / m a_{0}\right)^{1 / 2}$.

The potential $V(r)$ defined in this way depends on $T$ through $K_{m}$, that is through the equilibrium value of $N^{\prime}$. Since $N^{\prime}$ decreases with $T$ because of the corresponding increase of $\xi$, that is of the parameter describing the strength of dynamical correlations, we ultimately attribute the decrease of $V(r)$ to an increase with temperature of these correlations or, equivalently, of the probability rate for a hard core collision. The same conclusion was independently reached before, reasoning on the scattering processes produced by $V(r)$. This proves the consistency of a simple description in terms of a temperature dependent effective potential.

The oscillators appearing in $H^{0}$, being dynamically independent, cannot reach thermal equilibrium or exchange correlations with the environment. This shows that $H^{0}$ alone cannot describe the real liquid. Such a result is not surprising because a potential like $V(r)$ represents a long range interaction (see sect. 7), so that $H^{0}$ refers to a liquid deprived of the short range part, $V_{\mathrm{hc}}(r)$, of the pair potential. It is reasonable to treat precisely this part as the origin of the scattering processes which are responsible for the attainment of thermal equilibrium. This amounts to assuming that the Hamiltonian describing the real liquid differs from $H^{0}$ by a perturbation term $H_{p}=$ $(1 / 2) \Sigma_{i} \Sigma_{j \neq i} V_{\mathrm{hc}}\left(\boldsymbol{r}_{i}-\boldsymbol{r}_{j}\right)$, which plays the same role as the crystal anharmonicity (responsible for the thermalization of the phonon field in a crystal lattice).

For the short range part of the potential we use the simple form $V_{\mathrm{hc}}(r)=A \delta(\boldsymbol{r})$. The justification of this form lies in the existence of a hard core interaction between atoms, for which an atom $a$ is never transparent to another atom $b$, however energetic this is. In other words, the total scattering cross section, as presented by $a$ to $b$, does not vanish even when the relative velocity of $b$ with respect to $a$ is sent to infinity. Such a condition is precisely satisfied by the above potential. It has to be pointed out that, while $V(r)$ depends on $N^{\prime}$ and, therefore, works as a temperature-dependent effective potential, the constant $A$ depends only on the properties of the atomic core, so that $A \delta(\boldsymbol{r})$ is independent of the thermodynamical state of the liquid. We conclude that the liquid can be described as a system of atoms embedded in a constant background potential $-u^{0}$ and interacting through an effective potential, or pseudopotential, of the form $\Phi(r)=V(r)+A \delta(r)$.

The modes described by $H^{0}$ have a physical meaning only if they are not appreciably affected by the perturbation $H_{p}$. This requires $H_{p}$ to be small. However, $H_{p}$ must also be sufficiently strong to produce an atomic mean free path of the order of $0.1 \AA$, 
as a typical result of the scattering processes occurring in a liquid metal (see sect.7). Owing to our choice of $V_{\mathrm{hc}}(r)$, things go precisely in this way. In fact it is a success of the theory to check, on the one hand, that the value of $A$ required to explain the experimental diffusivities is about three orders of magnitude lower than $V_{0}$, so that $H_{p}$ actually represents a very small perturbation; on the other hand, that the same value of $A$ is precisely the one required to produce a mean free path of the expected order.

From the point of view of the diffusing atom, the liquid structure is simply an obstacle to the possibility of moving as a free particle. The atom feels the presence of the structure only as a limitation to its free motion. This is in agreement with a theory where the mean free path is merely deduced from structural properties (see sect. 7). In the spirit of such a theory, we expect that our simple description of the liquid, being able to predict the correct order of magnitude for the mean free path, automatically accounts, in a correct way, for the liquid structure.

\section{4- Transport equation and variational procedure.}

Let us now add to the system a tracer particle (solute atom), represented by an isotope of the atoms belonging to the liquid (solvent atoms). Its interaction with these atoms must still be described by $\Phi(r)$ and we can easily treat, by perturbation theory, the scattering processes undergone by such a particle as a consequence of the above interaction. These processes play a basic role in the Boltzmann equation, which is used to describe the diffusion of the solute atoms through the host liquid. It is peculiar of this equation to label the particles by a wavevector, that is to classify their states as plane wave states. Therefore, in both the initial and the final state of a scattering process the colliding atoms will be treated as free particles.

If the solute atom undergoes a scattering process in which its momentum changes by $\hbar \boldsymbol{K}$, a corresponding change $-\hbar \boldsymbol{K}$ must be registered in the host liquid. This change can be directly conferred to the system of phonons associated to the $3 N^{\prime}$ collective modes (case 1) or to a single atom of the liquid (case 2). The two processes are in competition, with different probability weights. Case 1 requires the creation of a phonon in the mode $-\boldsymbol{K}$, or the annihilation of a phonon in the mode $\boldsymbol{K}$. Case 2 requires a scattering process in which a single solvent atom undergoes a change $-\boldsymbol{K}$ of its wavevector.

Since no phonons exist for $K>K_{m}$, it follows that the processes referring to case 1 are confined to values of $K$ falling in the range between 0 and $K_{m}$. The theory of these processes [1] concerns the scattering of a particle by a system carrying longitudinal waves, under the assumption that all the particles interact through a Fourier transformable potential $\Phi(r)$. The probability rate is found to be proportional to $\Phi_{K}{ }^{2}$, which in the above range is approximately $V_{0}{ }^{2}$. Processes of this kind were treated in the elastic approximation and shown to be negligible. In Appendix $C$ such a conclusion is confirmed as a result of a rigorous calculation, which does not depend on the approximation of elastic scattering. The main contribution to the diffusivity comes from the processes referring to case 2 . These are described by a probability rate which is still proportional to $\Phi_{K}^{2}=V_{K}^{2}+A^{2}+2 A V_{K}$ and then, since $A<<V_{0}$, to $\Phi_{0}{ }^{2} \cong$ $V_{0}{ }^{2}$ for $K \leq K_{m}$ and to $A^{2}$ for $K>K_{m}$. Such a probability rate was denoted by $P\left(\boldsymbol{q}, \boldsymbol{q}^{\prime}\right)$ and explicitly given in terms of the wavevectors describing the initial and the final state of the solute atom, namely $\boldsymbol{q}$ and $\boldsymbol{q}^{\prime}=\boldsymbol{q}+\boldsymbol{K}$. 
Following the notation used earlier [1], we will denote by indices $a$ and $b$ the physical properties related to the solute atoms and to the solvent atoms, respectively: for instance, the symbols $m_{a}$ and $m_{b}$ will be used for the corresponding masses. Let $\mathcal{N}_{a}$ be the number of solute atoms per unit volume. In the presence of a drift force $\boldsymbol{F}_{a}$ acting on the solute atoms we arrive at a steady state with a concentration gradient $\nabla \mathcal{N}_{a}$ parallel to $\boldsymbol{F}_{a}$. Let us put $\nabla \mathcal{N}_{a}-\left(k_{\mathrm{B}} T\right)^{-1} \mathcal{N}_{a} \boldsymbol{F}_{a}=X \boldsymbol{U}$, where $\boldsymbol{U}$ is a unit vector along the direction of $\boldsymbol{F}_{a}$, and denote by $n(\boldsymbol{q}) \mathrm{d}^{3} q$ the number of solute atoms per unit volume with wavevector in $\mathrm{d}^{3} q$. Let us also introduce the function $\psi_{q}$ defined by the relation

$$
n(\boldsymbol{q})=\left[\left(\lambda_{a} / \pi\right)^{3 / 2} \mathcal{N}_{a}+\psi_{\boldsymbol{q}}\right] \exp \left(-\lambda_{a} q^{2}\right)
$$

It was shown that $\psi_{q}$ satisfies the equation

$$
-\left(\lambda_{a} / \pi\right)^{3 / 2} X \Sigma_{\boldsymbol{q}}\left(\boldsymbol{V}_{\boldsymbol{q}} \cdot \boldsymbol{U}\right) \psi_{\boldsymbol{q}} \exp \left(-\lambda_{a} q^{2}\right)=(2 \Omega)^{-1} \Sigma_{\boldsymbol{q}} \Sigma_{\boldsymbol{q}^{\prime}}\left(\psi_{\boldsymbol{q}}-\psi_{\boldsymbol{q}^{\prime}}\right)^{2} S\left(\boldsymbol{q}, \boldsymbol{q}^{\prime}\right)
$$

where $\boldsymbol{V}_{\boldsymbol{q}}=\hbar \boldsymbol{q} / m_{a}$ and $\lambda_{a}=\hbar^{2} /\left(2 m_{a} k_{\mathrm{B}} T\right)$; the kernel $S\left(\boldsymbol{q}, \boldsymbol{q}^{\prime}\right)$ was explicitly given in terms of $P\left(\boldsymbol{q}, \boldsymbol{q}^{\prime}\right)$ and shown to be symmetric with respect to the exchange of $\boldsymbol{q}$ with $\boldsymbol{q}^{\prime}$.

We can find an unlimited number of solutions of equation (4) which, therefore, differently from the transport equation, does not define $\psi_{q}$ in a unique way. Among all these solutions, the one satisfying the transport equation can be shown to produce, owing to the symmetry of $S\left(\boldsymbol{q}, \boldsymbol{q}^{\prime}\right)$, the maximum value of the right hand side. Such a property is the origin of the variational procedure. One can choose for $\psi_{q}$ a trial function depending on a set of adjustable parameters and determine these parameters in such a way that the right hand side of equation (4) is a maximum, under the constraint represented by equation (4) itself.

It was also shown that the required solution of the transport equation must be of the form $G(u)(\boldsymbol{q} \cdot \boldsymbol{U})$, where $G(u)$ is a function of the dimensionless variable $u=\lambda_{a}{ }^{1 / 2} q$ and satisfies the integral equation

$$
\chi u=\int_{0}^{\infty} y B(y) \mathrm{d} y \int_{-1}^{1}[u G(u)-(u+y \xi) G(t)] \exp (-E) \mathrm{d} \xi
$$

Here $\chi$ denotes a temperature dependent constant, which was explicitly expressed in terms of the microscopic parameters of the liquid [9], and the following definitions are used :

$$
\begin{gathered}
t=\left(u^{2}+y^{2}+2 u y \xi\right)^{1 / 2} \\
E=\mu^{-1}[\mu u \xi+(1+\mu) y / 2]^{2}
\end{gathered}
$$

where $\mu=m_{b} / m_{a}, B(y)=\Phi_{K}{ }^{2} / \Phi_{0}{ }^{2}$, the symbol $\Phi_{K}$ denoting the Fourier coefficient of $\Phi(r)$, evaluated for $K=\lambda_{a}^{-1 / 2} y$. For our model potential $\Phi_{K}{ }^{2}$ is equal to $\Phi_{0}{ }^{2}$ for $K \leq K_{m}$ and to $A^{2}$ for $K>K_{m}$, so that $B(y)$ is unity for $y \leq y_{m}$ and $A^{2} / \Phi_{0}{ }^{2}$ for $y$ $>y_{m}$, where $y_{m}=\lambda_{a}^{1 / 2} K_{m}$. 
Of course, if we were able to solve equation (5) with respect to $G(u)$, we could avoid the use of equation (4) and, consequently, of the variational procedure. Since, however, the solution of equation (5) represents a tremendous analytical problem, the above procedure becomes necessary. In the quoted paper [1] it was adopted in connection with a trial function of the form

$$
G(u)=\alpha+\beta u^{3}
$$

Such a form, when used to calculate the isotope effect for self-diffusion, was not found to produce any appreciable change with respect to the result previously deduced from the simple trial function $G(u)=$ constant. This suggested the weak sensitivity of the theory to the particular choice of the trial function: in other words, the property that we want now to prove in a more rigorous way.

The cubic term in equation (8) was justified by exploring the asymptotic behaviour, for $u \rightarrow \infty$, of the unknown solution of equation (5). It has to be said that a rigorous analysis of this behaviour would require a discussion of some convergence problems which were not properly considered in Appendix C of the quoted paper. However, such a discussion will not be given here, because we will not use the asymptotic behaviour to determine $G(u)$. The presence of a factor $u^{4} \exp \left(-u^{2}\right)$, which is found to weight $G(u)$ in the integral expressing the current density of diffusing particles [1], shows that the knowledge of $G(u)$ for $u \rightarrow \infty$ is of little interest. Roughly, we can say that the values of $u$ contributing to the integral lie in the interval $0 \leq u \leq 3$, which approximately defines the width of the peak corresponding to the above factor. It follows that only the knowledge of $G(u)$ in this interval can be of real interest for the theory of diffusion. Let us note that in a finite interval any regular function is well approximated by a polynomial, so that the above arguments ultimately suggest a polynomial as a suitable representation of $G(u)$. This means that, if $G(u)$ is written in the form of a Mac Laurin's expansion

$$
G(u)=\Sigma_{j} \alpha_{j} u^{j}
$$

only a few terms of the series are expected to play an effective role in the theory.

Let us use the variational procedure to determine the coefficients $\alpha_{j}$. The corresponding form of equation (4) becomes a generalization of equation (42) of the quoted reference [1], so that, putting

$$
\begin{gathered}
T_{a b}=\left(16 \pi^{3} / 3\right)\left(m_{b} / 2 \pi k_{\mathrm{B}} T\right)^{1 / 2}\left(v \hbar^{2} \lambda_{a}^{7 / 2}\right)^{-1} \\
\rho_{a}=A^{2} /\left(\lambda_{a}{ }^{2} H\right) \\
H=\left(K_{m}{ }^{4} \Phi_{0}{ }^{2}\right) / 4
\end{gathered}
$$

and defining the dimensionless matrix elements

$$
R_{j k}=\int_{0}^{\infty} u^{j+k+2} \exp \left(-u^{2}\right) \mathrm{d} u \int_{-1}^{1}\left[1+(j k+j+k) \xi^{2}\right] \exp \left(-\mu u^{2} \xi^{2}\right) \mathrm{d} \xi
$$




$$
\begin{gathered}
S_{j k}=\int_{0}^{\infty} y^{3} \mathrm{~d} y \int_{0}^{\infty} u^{2} \exp \left(-u^{2}\right) \mathrm{d} u \int_{-1}^{1}\left\{t^{j+k}+u^{2} y^{-2}\left(t^{j}-u^{j}\right)\left(t^{k}-u^{k}\right)+\right. \\
\left.u y^{-1} \xi\left[t^{j}\left(t^{k}-u^{k}\right)+t^{k}\left(t^{j}-u^{j}\right)\right]\right\} \exp (-E) \mathrm{d} \xi
\end{gathered}
$$

one finds the equation

$$
-\left(X \hbar / m_{a} \lambda_{a}\right) \Sigma_{j}\left(\alpha_{j} / C_{j}\right)=(2 \pi)^{-3} T_{a b} H \lambda_{a}^{2} \Sigma_{j} \Sigma_{k} M_{j k} \alpha_{j} \alpha_{k}
$$

where $M_{j k}=R_{j k}+\rho_{a} S_{j k}, C_{j}=\Gamma(5 / 2) / \Gamma[(5+j) / 2]$ and $\Gamma$ denotes the Euler's function.

Let us briefly comment the approximation staying behind this result. The rigorous expression for $M_{j k}$, as resulting from the right hand side of equation (4), turns out to be proportional to a multiple integral having the form of equation (14), but containing a factor $B(y)$ in the integrand. Splitting the integral over $y$ into a first integral between 0 and $y_{m}$ and a second integral between $y_{m}$ and $\infty$, one easily finds the rigorous form of $R_{j k}$. This is $\left(4 / y_{m}{ }^{4}\right)\left[1-\left(A / \Phi_{0}\right)^{2}\right]\left(S^{\prime}\right)_{j k}$, where $\left(S^{\prime}\right)_{j k}$ corresponds to equation (14) when the first integration range is confined to the interval $0 \leq y \leq y_{m}$. A numerical evaluation of $\left(S^{\prime}\right)_{j k}$ leads to values of $R_{j k}$ which do not appreciably differ from those deducible through equation (13), the relative discrepancy being always contained within two parts over thousand. This is reasonable, since $y_{m}$ is very small (see sect. 6) and justifies the expansion for $y_{m} \rightarrow 0$ from which equation (13) was derived.

The maximization of the right hand side of equation (15), under the constraint represented by equation (15) itself, leads to a system of linear equations for the coefficients $\alpha_{j}$. In terms of the ratios $f_{j}=\alpha_{j} / \alpha_{0}$ this system turns out to be

$$
\Sigma_{k \neq 0}\left[M_{0 k}-C_{j} M_{j k}\right] f_{k}=C_{j} M_{0 j}-M_{00}
$$

When the $f_{j}$ 's have been obtained as a solution of the system of equations (16), equation (15) fixes unambiguously $\alpha_{0}$, so that $G(u)$ is completely determined. At this point, the calculation of the current density of solute atoms is straightforward and leads to the following expression for the diffusion coefficient of an isotope of species $a$, moving through a liquid composed of isotopes of species $b$ :

$$
D^{a / b}=3 \pi^{2} v \sqrt{2}\left(k_{\mathrm{B}} T\right)^{5 / 2} m_{b}^{-1 / 2} H^{-1} W\left(\mu, \rho_{a}\right)
$$

where

$$
W\left(\mu, \rho_{a}\right)=\left[\Sigma_{j}\left(f_{j} / C_{j}\right)\right]^{2} /\left[\Sigma_{j} \Sigma_{k} M_{j k} f_{j} f_{k}\right]
$$

The sums include the terms with $j=0$ and $k=0$, for which $C_{0}=1, f_{0}=1$.

In principle, the Mac Laurin expansion of $G(u)$, as given by equation (9), is extended to all the integer values of $j$ between 0 and $\infty$. Under these conditions equation (16) stands for a set of infinite equations in the unknowns $f_{k}$. Apparently, this represents a problem of insurmountable difficulty for the determination of $G(u)$. 
However, we can solve the problem by confining the expansion to a finite number of terms, namely to $j \leq J$, and determine the behaviour of the solution as a function of $J$. If this behaviour is found to be convergent, the required solution can be obtained through an extrapolation procedure for $J \rightarrow \infty$. Such a procedure will be applied in sect. 6 , where we will successively determine the $f_{k}$ 's for all the integer values of $J$ between 0 and 6 , using the matrix elements $R_{j k}$ and $S_{j k}$ given by Table 1.

\section{5- Use of the Enskog formula for the self-diffusion coefficient.}

To determine $\rho_{a}$, it is convenient to consider the case of self-diffusion, that is the case in which the solute atom has the same isotopic composition and, therefore, the same mass $m_{a}$, of the solvent atoms. The self-diffusion coefficient is then

$$
D^{a / a}=3 \pi^{2} v \sqrt{2}\left(k_{\mathrm{B}} T\right)^{5 / 2} m_{a}^{-1 / 2} H^{-1} W\left(1, \rho_{a}\right)
$$

In the limit $\rho_{a} \rightarrow 0$, that is $A \rightarrow 0$, the scattering processes of the solute atom are only due to $V(r)$. They correspond to values of $K$ in the interval $0 \leq K \leq K_{m}$, where $V_{K}$ is a constant approximately equal to $\Phi_{0}$ and represented by equation (2).

Since $K_{m}$ is much lower than the value of $K$ corresponding to the mean thermal energy, we are here in a limit where only low angle scattering processes are considered. These do not contribute to the atomic mean free path $l$, which is essentially explained in terms of the high $K$ scattering processes allowed by the short range part of the interaction (see sect. 7). Therefore, the limit $A \rightarrow 0$ corresponds to a liquid with $l \rightarrow \infty$, as shown by the inverse proportionality relation between $l$ and $A^{2}$. An approximation of this kind is precisely the one staying behind the Enskog formula for the self-diffusion coefficient of a liquid of rigid spheres. In terms of the diameter $\sigma$ of these spheres and of the packing fraction $\eta_{p}=\pi \sigma^{3} / 6 v$, the formula is $([10]$, p.163)

$$
\mathcal{D}^{a / a}=\frac{3}{8}\left(v / \sigma^{2}\right)[g(\sigma)]^{-1}\left(k_{\mathrm{B}} T / \pi m_{a}\right)^{1 / 2}
$$

where $g(\sigma)=\left(1-\eta_{p}\right)^{-3}\left(2-\eta_{p}\right) / 2$ and $\eta_{p}$ is linked to $a_{0}$ through the relation [11]

$$
a_{0}=\left(1-\eta_{p}\right)^{4}\left(1+2 \eta_{p}\right)^{-2}
$$

Feinauer et al. [4], using a rigid sphere model for liquid sodium, showed that a proper treatment of correlation effects must be invoked to explain the strong rise with $T$ of the observed diffusivity, in striking contrast with the simple $T^{1 / 2}$ law predicted by the Enskog formula. This is not surprising, since the only correlation accounted for by equation (20) is the one described by $g(\sigma)$, a parameter strictly connected with the static structure factor, while correlations between particle velocities are disregarded. Thus the use of the above equation implies a temperature for which the effect of dynamical correlations is negligible. Since dynamical correlations increase with $T$, one deduces that, if such a temperature exists, it must necessarily be a low temperature. The lowest temperature at disposal, $T_{M}$, corresponds to the melting point: the final question is, therefore, if at $T=T_{M}$ the diffusivity can be simply expressed in 
terms of static correlation parameters, as required by the Enskog formula. A positive answer to this question would justify the use of equation (20) in the limit $l \rightarrow \infty$ at the melting point.

The answer is suggested by the structure of equation (19) for $\rho_{a}=0$, which according to the present theory expresses the diffusivity in the same limit. Here all the correlations are concealed in $H$, that is in $K_{m}$ or, as shown by equation (1), in $N^{\prime}$. In the expression deduced earlier for $N^{\prime}$ [2], the only parameter describing dynamical correlations is $\xi$, while the other parameters, like $\delta U_{\mathrm{s}}, \delta S_{\mathrm{s}}$ and $a_{0}$, refer to correlations existing in the static structure. Thus the absence of dynamical correlations, as required to justify the Enskog formula, amounts to assuming that $\xi$ can be approximately neglected at the melting point. The validity of this assumption is demonstrated by the calculations performed in sect. 8 of the quoted paper. In fact, only if $\xi$ is assumed to be very close to zero at $T=T_{M}$, it is possible to explain, in a consistent way, the temperature dependence of the self diffusion coefficients. Such a result represents a strong support in favour of the use of the Enskog formula at the melting point.

As a conclusion, at the above temperature, one is allowed to identify the right hand side of equation (20) with the expression provided by equation (19) for $\rho_{a}=0$. The expressions to be identified derive from two independent models, based on different interatomic potentials. The proposed identification is equivalent to assuming that both the models are good. The legitimacy of such an assumption is automatically justified by the value of $N^{\prime} / N$ to which the identification gives rise: as we will show in sect.6, this value turns out to be fully consistent with the upper limit predicted in sect. 2 .

\section{6- A convergent approach to the solution of the transport equation.}

Let us then put $\rho_{a}=0$ in equation (19) and identify the corresponding expression with the Enskog formula at the melting point. Using the index $M$ for any quantity referring to $T_{M}$ and, therefore, introducing the symbol $\mathcal{D}_{M}{ }^{a / a}$ to denote the expression provided by equation (20) for this temperature, we will impose the condition

$$
3 \pi^{2} v_{M} \sqrt{2}\left(k_{\mathrm{B}} T_{M}\right)^{5 / 2} m_{a}^{-1 / 2} W(1,0) / H_{M}=\mathcal{D}_{M}^{a / a}
$$

For consistency, owing to the absence of dynamical correlations in $\mathcal{D}_{M}{ }^{a / a}$, all the parameters appearing on the left hand side of equation (22) must be independent of $\xi$. Such a condition is automatically satisfied because we have imposed $\xi=0$ at the melting point, so that at this temperature the free energy of the liquid does not contain $\xi$. It follows that both $N^{\prime} / N$ (which enters the expression for $H_{M}$ ) and $T_{M}$ (which is determined by equating the Gibbs free energies of the liquid and the solid phase) are independent of the above variable, as expected.

As a consequence of equation (22), the general expression for $D^{a / b}$ is

$$
D^{a / b}=\mathcal{D}_{M}^{a / a}\left(v_{T} / v_{M}\right)\left(T / T_{M}\right)^{5 / 2}\left(m_{a} / m_{b}\right)^{1 / 2}\left(H_{M} / H_{T}\right) W\left[\mu, \rho_{a}(T)\right] / W(1,0)
$$

Let us first apply the above formula to the self-diffusion of ${ }^{7} \mathrm{Li}$ at the melting point $\left(T_{M}=454 \mathrm{~K}\right)$. In this case $a_{0}=0.031, \eta=0.428, v_{M}=22.72 \AA^{3}, \sigma=2.648 \AA$, so that, from equation $(20), \mathcal{D}_{M}^{7 / 7}=1.194 \cdot 10^{-8} \mathrm{~m}^{2} \mathrm{~s}^{-1}$. 
For a given choice of $J$, the degree of the polynomial by which we represent $G(u)$, we can evaluate $W(1,0)$ and use equation (22) to deduce $H_{M}$. More precisely, confusing $\Phi_{0}$ with $V_{0}$ and combining equations (12) and (2), we can solve equation (16) for $\rho_{a}=0$, calculate the corresponding value of $W(1,0)$ and determine $K_{m}$ at the melting point. The results are displayed in Table 2 , which provides both $W(1,0)$ and $y_{m}=\lambda_{a}^{1 / 2} K_{m}$ for all the values of $J$ between 0 and 6 . For $J=3$ we see that $K_{m}$ has already reached its asymptotic value, which turns out to be $0.542 \AA^{-1}$. Owing to equation (1), this implies $N^{\prime} / N=2.04 \cdot 10^{-2}$, in full agreement with the result obtained earlier [2] and with the upper limit deduced in sect. 2. At this point equation (23), written for $b=a, T=T_{M}$, provides, for the self-diffusion coefficient of ${ }^{7} \mathrm{Li}$ at this temperature, the expression

$$
D_{M}^{7 / 7}=\mathcal{D}_{M}{ }^{7 / 7} W\left(1, \rho_{7, M}\right) / W(1,0)
$$

Here we have used the notation $\rho_{\beta, M}=\rho_{\beta}\left(T_{M}\right)$, where $\beta$ is an index running over the two values 6 and 7 and referring to ${ }^{6} \mathrm{Li}$ and ${ }^{7} \mathrm{Li}$, respectively.

Using for the left hand side of equation (24) the experimental value $5.64 \cdot 10^{-9} \mathrm{~m}^{2} \mathrm{~s}^{-1}$ [3], we can solve this equation with respect to $\rho_{7, M}$. This is possible through a computer programme which, for any value of $\rho_{7, M}$, solves the system of equations (16) with respect to the $f_{k}$ 's and calculates, through equation (18), the corresponding value of $W\left(1, \rho_{7, M}\right)$. The input value of $\rho_{7, M}$ is varied until $W\left(1, \rho_{7, M}\right)$ is found to satisfy equation (24). The value representing the solution is given in Table 2; the table also provides the corresponding values of the $f_{k}$ 's . These values determine, apart from a constant factor, the dependence on $u$ of the trial function, namely of the function $G(u)=\alpha_{0} \Sigma_{j} f_{j} u^{j}$. It is interesting to see how, by increasing $J$, we improve the approximation by which this function satisfies the integral equation (5). When inserted into the right hand side of this equation, the true $G(u)$ is expected to provide a linear behaviour vs $u$, as required by the left hand side. For a given $J$, let us introduce a function $S_{J}(u)$, defined by the right hand side of equation (5) when $G(u)$ is identified with $h_{J}(u)=\Sigma_{j} f_{j} u^{j}$, the sum being extended from $j=0$ to $j=J$. A plot of $S_{J}$ vs $u$ allows us to judge the reliability of the trial function by observing the deviation from linearity of the corresponding behaviour.

$S_{J}(u)$ can be split into a first integral between 0 and $y_{m}$, with $B(y)=1-\left(A^{2} / \Phi_{0}{ }^{2}\right)$, and a second integral, between 0 and $\infty$, with $B(y)=A^{2} / \Phi_{0}^{2}$. Now, using equations (11) and (12), we find that the ratio

$$
A^{2} / \Phi_{0}^{2}=\frac{1}{4} y_{m}^{4} \rho_{7, M}
$$

turns out to be smaller than $10^{-6}$ (see Table 2 ), so that we can simply put $B(y)=1$ in the first integral.

In order to present in the same figure the plots corresponding to different values of $J$, it is convenient to avoid confusions due to overlapping of the different curves. For this reason in fig. 1 we plot the functions $L_{J}(u)=J+S_{J}(u)$, where the additive constant $J$ is precisely introduced to separate the curves. The number associated to each curve represents the corresponding value of $J$. The gain in linearity, as produced by an 
increase of $J$, is evident. It is also possible to see how such a gain is accompanied by a progressive extension of the range where the behaviour is approximately linear; in fact, in going from $J=3$ to $J=6$, the upper limit of this range approximately increases from $u=3$ to $u=3.5$.

\section{7- Interpretation of the experimental data for liquid lithium.}

It is plausible to guess that an extension of the present numerical analysis to larger and larger values of $J$ would produce the required linear behaviour in the full range of the $u$-axis. There is, however, a good reason to avoid the supplementary work required by this procedure. This is connected with the fact that the diffusion coefficient, in spite of the presence of the $f_{k}$ 's in its expression given by equations (23) and (18), depends very weakly on the order $J$ of the polynomial by which the trial function is represented. To clarify this point, let us note that the aim of a theory of diffusion is not represented by the analytical form of $G(u)$; rather, it consists in specifying the dependence of $D^{a / b}$ on the physical properties of the host liquid and of the particles diffusing through it. Now, we can show that the form of this dependence changes with $J$, but in such a way that the change is confined to the lowest values of this parameter. In more precise terms, we can prove that, although for $J=2$ the trial function is still far from representing the true $G(u)$, all the numerical predictions of equation (23) at this order have already reached their asymptotic values, as shown by the fact that they do not appreciably differ from those corresponding to higher orders. To enter the details of the proof, we will discuss three predictions of the theory. These concern

a) the ratio $D^{6 / 6} / D^{7 / 7}$ at the melting point;

b) the ratio $D^{6 / 6}(T) / D^{6 / 6}\left(T_{M}\right)$, as deducible from the theory when the corresponding ratio $D^{7 / 7}(T) / D^{7 / 7}\left(T_{M}\right)$ is taken from experiment;

c) the values of $D^{6 / 7}$ and of $D^{7 / 6}$ at the melting point.

With reference to point $a$ ), one has simply to write equation (23) for $a=b=6$, in which case it provides

$$
D^{6 / 6}\left(T_{M}\right)=\mathcal{D}_{M}{ }^{6 / 6} W\left(1, \rho_{6, M}\right) / W(1,0)=\left(m_{7} / m_{6}\right)^{1 / 2} \mathcal{D}_{M}{ }^{7 / 7} W\left(1, \rho_{6, M}\right) / W(1,0)
$$

that is, owing to equation (23) itself, written for $a=b=7$,

$$
\left(D^{6 / 6} / D^{7 / 7}\right)_{M}=\left(m_{7} / m_{6}\right)^{1 / 2} W\left(1, \rho_{6, M}\right) / W\left(1, \rho_{7, M}\right)
$$

where

$$
\rho_{6, M}=\left(m_{6} / m_{7}\right)^{2} \rho_{7, M}
$$

With reference to point $b$ ), one obtains from equation (23)

$$
D^{\beta / \beta}(T) / D^{\beta / \beta}\left(T_{M}\right)=\left(v_{T} / v_{M}\right)\left(T / T_{M}\right)^{5 / 2}\left(H_{M} / H_{T}\right) W\left[1, \rho_{\beta}(T)\right] / W\left(1, \rho_{\beta, M}\right)
$$

where

$$
\rho_{\beta}(T)=\left(m_{\beta} / m_{7}\right)^{2}\left(T / T_{M}\right)^{2}\left(H_{M} / H_{T}\right) \rho_{7, M}
$$


For a given temperature $T$, the ratio $\Psi(T)=H_{T} / H_{M}$ can be immediately deduced from equation (29) written for $\beta=7$, when the left hand side of this equation is identified with the experimental ratio $D^{7 / 7}(T) / D^{7 / 7}\left(T_{M}\right)$. The resulting value of $\Psi(T)$ is then substituted into equation (29) written for $\beta=6$ and the ratio $D^{6 / 6}(T) / D^{6 / 6}\left(T_{M}\right)$ can be predicted.

Finally, with reference to point $c$ ), one deduces from equation (23), written for $a=$ 6 and $b=7$,

$$
\begin{gathered}
D^{6 / 7}\left(T_{M}\right)=\mathcal{D}_{M}{ }^{6 / 6}\left(m_{6} / m_{7}\right)^{1 / 2} W\left(m_{7} / m_{6}, \rho_{6, M}\right) / W(1,0)= \\
\mathcal{D}_{M}{ }^{7 / 7} W\left(m_{7} / m_{6}, \rho_{6, M}\right) / W(1,0)
\end{gathered}
$$

and from equation (23), written for $a=7$ and $b=6$,

$$
D^{7 / 6}\left(T_{M}\right)=\mathcal{D}_{M}{ }^{7 / 7}\left(m_{7} / m_{6}\right)^{1 / 2} W\left(m_{6} / m_{7}, \rho_{7, M}\right) / W(1,0)
$$

All the results of our predictions are contained in Table 3. The results referring to point $b$ ) are here presented in terms of the exponent $n$, defined by the equation

$$
D^{6 / 6}(T) / D^{6 / 6}\left(T_{M}\right)=\left(T / T_{M}\right)^{n}
$$

which was used by Feinauer et al. [3] to describe, in an empirical way, the observed behaviour of the diffusivity in the range between $T_{M}=454 \mathrm{~K}$ and $T_{1}=580 \mathrm{~K}$. To determine $n$, we applied the definition (33) to $T=T_{1}$, having deduced the corresponding theoretical value of the left hand side through the procedure previously discussed as a comment to equations (29) and (30). Such a procedure required the experimental ratio $D^{7 / 7}\left(T_{1}\right) / D^{7 / 7}\left(T_{M}\right)$, which was identified with $\left(T_{1} / T_{M}\right)^{2.37}$, according to the power law presented by the same authors. The thermal expansion coefficient $1.9 \cdot 10^{-4} \mathrm{~K}^{-1}$ was used to calculate the ratio $v_{T} / v_{M}$, as required by equation (29).

A first comment to Table 3 concerns the strong convergence of the variational procedure, for which, as previously anticipated, a $2^{\text {nd }}$ order polynomial of the form of equation (9) is sufficient to represent $G(u)$. This does not mean that, in order to obtain the mathematical solution of the transport equation, the approximation represented by the above polynomial is satisfactory: rather, it means that any improvement with respect to such an approximation is not necessary, for the purposes of a theory of diffusion. It is instructive, anyway, to see how a progressive increase of $J$ allows us to approach the true solution of the above equation. To this purpose, it is convenient to examine the curves reproducing the behaviour of the function $h_{J}(u)=$ $\Sigma_{j} f_{j} u^{j}$ for different values of $J$. In the interval $0 \leq u \leq 3$ the discrepancy between the curves corresponding to two successive values of $J$ is found to be strongly reduced by an increase of $J$. The maximum discrepancy, which amounts to about $100 \%$ for the couple of curves with $J=0$ and $J=1$ (not shown in fig. 2), reduces to only $3 \%$ for the couple defined by $J=5$ and $J=6$. The convergence properties of the variational procedure are clearly shown by the above figure, where, 
in fact, the curve corresponding to $J=6$ is found to lie inbetween the curves with $J$ $=4$ and $J=5$.

A further increase of $J$ is not expected to produce any substantial change of the curve in the above interval, so that here the sixth order polynomial can be considered as an excellent approximation of the true $G(u)$. Considerable changes for $J>6$ are only expected in the interval $u>3$ where, as a consequence, the determination of the true $G(u)$ remains an open problem. Since, however, the choice $J=2$ is already sufficient to warrant a reliable expression for $D^{a / b}$, the above problem is not of interest for a theory of diffusion. This is not surprising because, as already pointed out in sect. 4, the main contribution to the current density of diffusing particles is expected to be brought by the interval with $u<3$. The nice and remarkable result is that in this interval only a rough knowledge of $G(u)$ is required.

A second comment to Table 3 derives from a comparison with the experimental data. All the predictions of the Table are in striking agreement with the measurements of Feinauer et al.[3,4]. The consistency of our predictions with the experimental values $\left(D^{6 / 6} / D^{7 / 7}\right)_{M}=1.25$ and $n=2.68$, as given by these authors, was already stressed earlier [1]. We can now check the consistency of the theory with the measured interdiffusion coefficients $D^{6 / 7}$ and $D^{7 / 6}$. The experimental determination of these coefficients involves an extrapolation procedure, starting from the diffusivities of ${ }^{6} \mathrm{Li}$ and ${ }^{7} \mathrm{Li}$ in different mixtures of the two isotopes. Denoting by $D^{6}$ and $D^{7}$, respectively, the above diffusivities, and by $x_{7}$ the concentration of ${ }^{7} \mathrm{Li}$ in the mixture, we deduce that $D^{6 / 7}$ corresponds to the limit of $D^{6}$ for $x_{7} \rightarrow 1, D^{7 / 6}$ to the limit of $D^{7}$ for $x_{7} \rightarrow 0$. To obtain the above limits, Feinauer et al. $[3,4]$ made the reasonable assumption of a linear behaviour of the diffusivities in the whole range of $x_{7}$. This assumption is obviously justified by the small change presented by the diffusivities in going from $x_{7}=0$ to $x_{7}=1$. The same assumption can now be joined with our theoretical predictions of $D^{6 / 7}, D^{7 / 6}$ and $D^{6 / 6}$, in which case it allows us to predict the dependence of $D^{6}$ and $D^{7}$ on $x_{7}$ and then to obtain, after a comparison with the experimental dependence, a further check of the theory.

Fig. 3 shows the experimental values of $D^{6}$ (full squares) and of $D^{7}$ (triangles) at the melting point, with the uncertainties assigned to these values by the above authors. The corresponding theoretical behaviours, predicted through the values $D^{6 / 7}$ $=6.38, D^{7 / 6}=6.29$ and $D^{6 / 6}=1.244 \times 5.64=7.02$ (in units $10^{-9} \mathrm{~m}^{2} \mathrm{~s}^{-1}$, see Table 3 ), are represented by the straight lines $a$ and $b$, respectively. We see that the whole set of data is explained by the theory within the experimental uncertainties. The significance of this result lies in the fact that we have not introduced any adjustable parameter, having only imposed the value of $D^{7 / 7}$ at $T_{M}$, which was identified with the extrapolated value, $5.64 \cdot 10^{-9} \mathrm{~m}^{2} \mathrm{~s}^{-1}$, as given by the same authors in the quoted papers $[3,4]$. The consistency of the theory is enhanced by the value of the atomic mean free path deriving from such identification: from the resulting value of $A^{2} / \Phi_{0}{ }^{2}$, as given by Table 2 , taking $\Phi_{0} \cong V_{0}$ from equation (2) and using, for a liquid with atomic mass $m$, the expression deduced earlier [1] for the mean free path $l$, namely

$$
l=7.678 v \hbar^{4}(m A)^{-2}
$$


we obtain, for ${ }^{7} \mathrm{Li}$ at the melting point, $l=1.51 \cdot 10^{-1} \AA$, which is of the expected order of magnitude.

Faber ([10], p.105) pictured a solid metal at the melting point as a regular array of rigid spheres and assumed each sphere to move freely inside its Voronoi polyhedron (as obtained by drawing planes to bisect the lines joining the sphere with its nearest neighbours). For such a model he deduced an atomic r.m.s. displacement given by $X$ $=0.11 R_{A}$, where $R_{A}=(3 v / 4 \pi)^{1 / 3}$. If one adopts the same model for the liquid phase, the Voronoi polyhedron becomes the cage of nearest neighbours and $X$ can be identified with the atomic mean free path, which in this way, for ${ }^{7} \mathrm{Li}$ at the melting point, turns out to be $0.19 \AA$. In spite of the crudeness of the model, this number is certainly good to represent the order of magnitude expected for $l$. Its close agreement with $0.15 \AA$, the value inferred from our interpretation of diffusion data, is a strong support in favour of the reliability of the whole theory.

Equation (34) amounts to assuming that only the short range part of the pair potential plays a role in the determination of the mean free path. We can easily understand why $V(r)$, which is responsible for the classical contribution to the diffusion coefficient, is not called into play. Since the Fourier coefficients $V_{K}$ are confined to the small range $0 \leq K \leq K_{m}$, it follows that $V(r)$ must be proportional to $\mathcal{G}\left(K_{m} r\right)$, where $\mathcal{G}(x)=3(\sin x-x \cos x) / x^{3}$. The first zero of $\mathcal{G}(x)$ is about 4.5 , so that the range of $V(r)$ is approximately defined by $r_{0}=4.5 / K_{m} \cong 9 \AA$; this is about 60 times the value of $l$ determined by the point-like potential $A \delta(\boldsymbol{r})$. One concludes that $V(r)$ is a long range function or, more precisely, a slowly varying function in an interval of length $l$. Let us then start from a liquid where only the point-like potential is active and consider an atom which, under these conditions, behaves as a free particle along a path of the above length. If we switch on $V(r)$, the weak variation of $V(r)$ in this path corresponds to a very small force acting on the atom. The only result of this soft force is a small bending of the trajectory of the atom inbetween two successive collisions, without introducing any additional collision centre. Therefore, the mean free path remains unchanged.

The above conclusions are supported by energy considerations. If the soft force has a negligible effect on the trajectories of the particles, it has to be expected that the mean work done by this force along a path of length $l$ is much smaller than the mean kinetic energy per atom. Such a guess is easily checked. In fact, the work is represented by the change of the soft potential over a distance $l$, that is, on the average, by $\left[V(0) / r_{0}\right] l$, where $V(0)=3 N^{\prime} \Omega^{-1} V_{0}$ is the limit of $V(r)$ for $r \rightarrow 0$. Owing to equation (2), the ratio of this work to $3 k_{\mathrm{B}} T / 2$ is $2\left(a_{0}^{-1}-1\right)\left(N^{\prime} / N\right)\left(l / r_{0}\right)$. As expected, this is a small number, amounting to about $2 \cdot 10^{-2}$ for lithium at the melting point.

To summarize, we can say that the behaviour of an atom is quite similar to that of a small charged sphere, moving on a plate which carries a distribution of nails perpendicular to the plate. The mean free path of the sphere is totally determined by this distribution, independently of a small external electric field which can be applied to the sphere. The only effect of this field is that the sphere does not proceed along a straight line in the time interval between two successive collisions with the nails. 


\section{8- Limits of the numerical procedure.}

In order to obtain the coefficients $S_{j k}$ of Table 1, the integrations over $y, u$ and $\xi$ required by equation (14) were performed numerically. To this purpose the integration paths were subdivided into intervals of width $p_{1}=0.05, p_{2}=0.05$ and $p_{3}$ $=0.005$, respectively. Where a comparison with rigorous analytical expressions is possible (for instance, in the case of $S_{00}$ [1]), such a choice proves to be fully satisfactory, the maximum error being contained within a few parts over thousand. Unexpectedly, an improvement in the determination of the $S_{j k}$ 's (which mainly depends on a reduction of $p_{3}$ ) does not produce a gain in our knowledge of $G(u)$, leading to numerical instabilities in the solution of equation (16). When $p_{3}$ is reduced to 0.0025 , the new values of the $S_{j k}$ 's are of course very close to the corresponding values of Table 1 , the corrections being of the order of $0.1 \%$. Nevertheless, if under these conditions equation (16) is solved for $J \geq 5$, the resulting $f_{j}$ 's turn out to be strongly oscillating functions of $\rho_{a}$. More precisely, very small changes of $\rho_{a}$ (of the order of $10^{-5}$ ) engendre fluctuations in the form of the curve representing $h_{J}(u)$. These fluctuations mean that in the above case the polynomial representation of the trial function is not fully adequate to provide a reliable approach to the true $G(u)$. In principle, one could vary $\rho_{a}$ over an interval containing many oscillations and perform a corresponding average of $h_{J}(u)$. Such a procedure would eliminate the fluctuations, but would also introduce some errors, owing to the ambiguities connected with an exact definition of the same interval. Thus, in the mentioned case, the only reliable information on $G(u)$ derives from the solutions of equation (16) for $J \leq 4$, which do not present the oscillating behaviour. The conclusion is that it is certainly better to work with the values of the $S_{j k}$ 's given in Table 1 , for which, at least up to $J=6$, no numerical instability of the solutions is observed.

We note, however, that such a conclusion is of mathematical, not a physical interest. It must be taken into account if we wish to obtain the best numerical approach to the solution of the integral equation (5); it has no weight if our aim is to predict the isotope effects, which, in fact, are almost insensitive to the oscillating behaviour of the $f_{j}\left(\rho_{a}\right)$ 's. If, for $J \geq 5$, these coefficients are inserted into equations (18) and (23), the resulting diffusion coefficients do not show any significant oscillation when $\rho_{a}$ is varied in the neighbourhhod of the values appearing in Table 2 . There is only a weak residual fluctuation (of the order of a few parts over thousand) which can be averaged without appreciable error. As a consequence we find again (without significant changes) all the numerical values of Table 3 . We deduce that the transition of $p_{3}$ from 0.005 to 0.0025 does not alter the predictions of the theory. This result is in agreement with any reasonable expectation and also confirms the weak dependence of the theory on the form of the trial function by which the solution of the transport equation is approximated.

\section{9- Final conclusions.}

The quantum mechanical theory of diffusion, as developed earlier for liquid metals $[1,2]$, lies on a pseudopotential formulation, which now finds its full and final justification in the discussion presented in sects. 2 and 3. The simple form of pseudopotential adopted in the quoted papers is shown to represent a powerful tool to 
explain all the experimental data of Feinauer, Majer and Seeger on liquid lithium $[3,4]$. The attention of our previous work was essentially confined to the self diffusion coefficients of the two lithium isotopes, while the possibility of predicting the absolute values of the two interdiffusion coefficients $D^{6 / 7}$ and $D^{7 / 6}$ was overlooked. The close agreement between the predicted and the measured values of these coefficients, as is now found in sect. 7, represents a further and important support to the whole formulation. We note that the theory is also able to predict the temperature dependence of the same coefficients: using the formulae contained in the above section, it would be easy to obtain, in units $10^{-9} \mathrm{~m}^{2} \mathrm{~s}^{-1}$, the values $D^{6 / 7}=$ 11.77 and $D^{7 / 6}=11.74$ at $580 \mathrm{~K}$. These correspond to power laws characterized by exponents $n=2.50$ and $n=2.55$, respectively. Unfortunately, the above predictions cannot find an experimental support in the work of the mentioned authors, who confined their investigations to the melting point. The obvious conclusion is that new measurements of interdiffusion coefficients, giving information on the corresponding temperature dependence, would be welcome. More generally, there is an interest for an extension of the temperature range defining the observed behaviour of all the coefficients, so as to allow a full comparison between theory and experiment at temperatures significantly higher than $580 \mathrm{~K}$.

\section{Appendix A.}

As shown earlier [2], the result of the minimization of the free energy is an expression of $x=N^{\prime} / N$ in terms of $\xi$, namely of the form $x=f(\xi)$ (see equation (49) of the above reference), where the probability $p=\xi / v$ refers to a process in which a liquid-like atom becomes a Mott oscillator, in the field produced by a solidlike atom of frequency $\omega_{m}=s K_{m}=\left(k_{\mathrm{B}} T / m a_{0}\right)^{1 / 2} K_{m}$. Thus $\xi$ depends on $\omega_{m}$, that is on $K_{m}$, which in turn depends on $x$ through equation (1). The conclusion is that $x=f(\xi)$ defines $x$ only implicitly: it must be considered as an equation to be solved with respect to $x$. For a liquid described by a classical continuum, the solution will be a dimensionless quantity depending on the viscosity $\eta_{v}$ (which is expected to determine $p$ ), on the atomic volume $v$ and on the atomic mass $m$, and finally on the set $q$ of all the microscopic parameters which are necessary to describe the structure change required to create the Mott oscillator. By definition, the parameters concealed in $q$ are independent of $m$. We expect $x$ to be expressible in terms of quantities like $\alpha=\eta v^{2 / 3}\left(m k_{\mathrm{B}} T\right)^{-1 / 2}$ and $\beta=\eta v^{2 / 3}(m \varepsilon)^{-1 / 2}$, where $\varepsilon$ is any energy term belonging to the set $q$ : in fact, $\alpha$ and $\beta$ provide the only possible expressions for dimensionless quantities containing $\eta_{v}$. For the classical fluid we are considering, both these expressions are independent of $m$ : this follows from a well known result of statistical mechanics, according to which $\eta_{v}$ is proportional to $m^{1 / 2}$ ([9], p. 165). The final result is that $x$ does not depend on the mass of the atoms and, owing to equation (1), the same property holds for $K_{m}$, as required by the theory outlined in sect. 2 .

\section{Appendix B.}

For any function $f(r)$, the Fourier coefficients $f_{k}$ will be defined by the relations 


$$
f(r)=\Omega^{-1} \Sigma_{k} f_{k} \exp (i \boldsymbol{k} \cdot \boldsymbol{r}), \quad f_{k}=\int f(r) \exp (-i \boldsymbol{k} \cdot \boldsymbol{r}) \mathrm{d}^{3} r
$$

$\Omega$ being the volume of the liquid.

Let us then consider the liquid described by the Hamiltonian $H^{0}$ (see sect. 3), corresponding to a system of $N$ atoms embedded in an external potential $-u^{0}$ and mutually interacting through the soft potential $V(r)$. In such a case the mean energy of interaction can be written

$$
\begin{aligned}
U_{i n t}= & \frac{1}{2} \Sigma_{i} \Sigma_{j \neq i} V\left(\boldsymbol{r}_{i}-\boldsymbol{r}_{j}\right)=\frac{1}{2} \Omega^{-1} \Sigma_{i} \Sigma_{j \neq i} \Sigma_{k} V_{k} \exp \left[i \boldsymbol{k} \cdot\left(\boldsymbol{r}_{i}-\boldsymbol{r}_{j}\right)\right]= \\
& \frac{1}{2} \Omega^{-1} \Sigma_{i} \Sigma_{k} V_{k} \int(N / \Omega)[1+\sigma(\rho)] \exp (i \boldsymbol{k} \cdot \rho) \mathrm{d}^{3} \rho
\end{aligned}
$$

where the atomic distribution in the neighbourhood of the $i^{\text {th }}$ atom has been expressed in terms of the pair correlation function $\sigma(r)$. As a consequence the total mean potential energy can be written

$$
U=\frac{1}{2}\left(N^{2} / \Omega\right)\left[V_{O}+\Omega^{-1} \Sigma_{k} V_{k} \sigma_{k}\right]-N u^{0}
$$

where $\sigma_{k}$ is linked to the structure factor $a_{k}$ by the relation $a_{k}=1+N \Omega^{-1} \sigma_{k}$ and the sum is confined to the wavevectors of the collective modes, because $V_{k}=0$ for any other wavevector. It follows that the term containing this sum, which turns out to be $3 V_{0}\left(a_{0}-1\right)\left(N^{\prime} / N\right)$, brings a negligible contribution to the square bracket.

We note that $U$, for consistency of the model, must represent the experimental mean potential energy of the liquid, as deducible from specific heat data. These data suggest a total energy of the form $\alpha N k_{\mathrm{B}} T$, where $\alpha$ is 3.35 for liquid lithium [2] and would be 3 for a liquid obeying the Dulong and Petit law. Subtracting the mean kinetic energy we are left with a term $(\alpha-3 / 2) N k_{\mathrm{B}} T$, which has to be identified with $U$. Such identification provides the value of $u^{0}$; using equation (2) and neglecting the small term with $N^{\prime} / N$ in equation (B3), one finds

$$
u^{0}=\frac{1}{2}\left(a_{0}^{-1}-1-2 \alpha+3\right) k_{\mathrm{B}} T
$$

The meaning of a background potential proportional to $T$, as predicted by equation (B4), can be easily understood in the frame of the Percus and Yevick theory [7]. In such a theory the total mean potential energy is simply represented by equation (B3), the sum over $\boldsymbol{k}$ being now extended to $3 N$ wavevectors, and $V_{k}$ expressed in terms of the structure factor $a_{k}$ by the Ascarelli, Harrison and Paskin relation. In this case $V_{0}$ is still given by equation (2) and the result is

$$
U=\frac{1}{2}\left(N^{2} / \Omega\right) V_{0}-N\left[\frac{1}{2} N^{-1} \Sigma_{k \neq 0}\left(a_{k}-1\right)^{2} a_{k}^{-1}\right] k_{\mathrm{B}} T
$$


A comparison between equation (B5) and equation (B3) (where the second term in the square bracket can be neglected), shows precisely the existence of a temperature dependent background potential $u^{0}$. It also shows that $u^{0}$ accounts for the presence of a liquid structure: in fact it would vanish for a structureless liquid, corresponding to $a_{k}=1$.

\section{Appendix C.}

To account for the scattering processes due to the interaction between the diffusing particle and the phonon field, we must add to the right hand side of equation (4) a term, say $\mathcal{R}$, containing the probability rate for the above processes. Owing to the definition of function $\psi_{q}$ (see sect. 4) and to equations (D2) and (D3) of ref. [1], this term in the high temperature limit is obtained through the substitution of $S\left(\boldsymbol{q}, \boldsymbol{q}^{\prime}\right)$ by

$$
\begin{gathered}
\mathcal{L}\left(\boldsymbol{q}, \boldsymbol{q}^{\prime}\right)=\left(\pi \Phi_{0}^{2} / v m_{b} s^{2} \hbar \theta\right) \Sigma_{\boldsymbol{Q}}\left[\delta\left(\varepsilon_{\boldsymbol{q}}-\varepsilon_{\boldsymbol{q}^{\prime}}-\hbar \omega_{\boldsymbol{Q}}\right) \exp \left(-\theta \varepsilon_{\boldsymbol{q}^{\prime}}\right) \delta(\boldsymbol{K},-\boldsymbol{Q})+\right. \\
\left.\delta\left(\varepsilon_{\boldsymbol{q}}-\varepsilon_{\boldsymbol{q}^{\prime}}+\hbar \omega_{\boldsymbol{Q}}\right) \exp \left(-\theta \varepsilon_{\boldsymbol{q}}\right) \delta(\boldsymbol{K}, \boldsymbol{Q})\right]
\end{gathered}
$$

where the sum is over all the phonon wavevectors $\boldsymbol{Q}$ and $\boldsymbol{K}=\boldsymbol{q}^{\prime}-\boldsymbol{q}, \theta=1 / k_{\mathrm{B}} T$, $\varepsilon_{q}=\hbar^{2} q^{2} / 2 m_{a}, \omega_{Q}=s Q$.

If $\mathcal{R}$ is small, we expect $\psi_{q}$ to be very close to the unperturbed trial function, as discussed in sect. 4 . To the first order of a perturbation theory, $R$ is simply obtained by substituting $\mathcal{L}\left(\boldsymbol{q}, \boldsymbol{q}^{\prime}\right)$ to $S\left(\boldsymbol{q}, \boldsymbol{q}^{\prime}\right)$ and leaving $\psi_{\boldsymbol{q}}$ unchanged. To estimate the order of magnitude of $\mathcal{R}$, it is sufficient to represent $\psi_{\boldsymbol{q}}$ by $\alpha(\boldsymbol{q} \cdot \boldsymbol{U})$, where $\alpha$ is a constant. Writing the sum over $\boldsymbol{q}^{\prime}$ as a sum over $\boldsymbol{K}$ and transforming both the sums over $\boldsymbol{q}$ and over $\boldsymbol{K}$ into integrals, one can rigorously perform all the integrations through the procedure outlined in sect. 5 of ref. [1]. Introducing the definitions $k_{0}=2 m_{a} s / \hbar$ and $\varepsilon_{0}=m_{a} s^{2} /\left(2 k_{\mathrm{B}} T\right)$, one finds

$$
\begin{gathered}
\mathcal{R}=\Omega \alpha^{2} m_{a}^{2} \Phi_{0}^{2}\left(24 \pi^{3} \hbar^{5} v m_{b} s^{2} \theta^{2}\right)^{-1} \\
\int_{0}^{K_{m}} K^{3} \exp \left[-\varepsilon_{0}\left(1-k_{0}{ }^{-1} K\right)^{2}\right] \mathrm{d} K
\end{gathered}
$$

One can now take the ratio of $\mathcal{R}$ to the unperturbed value of the right hand side of equation (4), as deducible from sect. 5 of the quoted reference. Such a ratio measures the weight of the above processes with respect to the leading contribution of the processes considered in sect. 4 . Since $k_{0} \gg>K_{m}$ the resulting ratio is approximately

$$
\rho=\frac{1}{2}\left(\pi m_{a} / m_{b}^{3}\right)^{1 / 2}\left(k_{\mathrm{B}} T / s^{2}\right)\left(0.63+1.25 \gamma^{2}\right)^{-1} \exp \left(-\varepsilon_{0}\right)
$$

where

$$
\gamma=4 m_{a} k_{\mathrm{B}} T\left(\hbar K_{m}\right)^{-2}\left(A / \Phi_{0}\right)
$$


Owing to the large value of $\varepsilon_{0}$ the ratio represented by equation (C3) turns out to be completely negligible. Referring for instance to the case of interest for selfdiffusion $\left(m_{a}=m_{b}\right)$ and using the values of $K_{m}$ and $A / \Phi_{0}$ previously determined for lithium, we find, for this metal at the melting point, $\gamma=0.65, \varepsilon_{0}=16.1$ and then $\rho=2.5 \cdot 10^{-9}$.

\section{REFERENCES}

[1] M. Omini, Phil. Mag. 861643 (2006).

[2] M. Omini, Phil. Mag. 875249 (2007).

[3] A. Feinauer, G. Majer and A. Seeger, J. Phys. Condensed Matter 6355 (1994).

[4] A. Feinauer, G. Majer and A. Seeger, Defect Diffusion Forum 143-147 881 (1997).

[5] M. Shimoji, Liquid Metals (Academic Press, New York, 1977).

[6] N. F. Mott, Proc. Roy. Soc. 146465 (1934).

[7] J. K. Percus and G. J. Yevick, Pys. Rev. 1101 (1958).

[8] P. Ascarelli, R. J. Harrison and A. Paskin, Adv. Phys. 16717 (1967).

[9] M. Omini, Phil. Mag. A 54561 (1986).

[10] T. E. Faber, An Introduction to the Theory of Liquid Metals (Cambridge Univ. Press, 1972).

[11] N. W. Ashcroft and J. Leckner, Phys. Rev. 14583 (1966). 


\begin{tabular}{|llll|llll|}
\hline & $\mu=1$ & $\mu=7 / 6$ & $\mu=6 / 7$ & & $\mu=1$ & $\mu=7 / 6$ & $\mu=6 / 7$ \\
$R_{00}$ & 0.6267 & 0.6021 & 0.6503 & $S_{00}$ & 1.2456 & 1.3874 & 1.1017 \\
$R_{11}$ & 1.2533 & 1.1578 & 1.3507 & $S_{11}$ & 3.5078 & 3.9119 & 3.1014 \\
$R_{22}$ & 3.8774 & 3.4592 & 4.3242 & $S_{22}$ & 13.960 & 15.470 & 12.429 \\
$R_{33}$ & 15.627 & 13.564 & 17.917 & $S_{33}$ & 71.000 & 78.123 & 63.737 \\
$R_{44}$ & 77.277 & 65.714 & 90.519 & $S_{44}$ & 436.87 & 477.54 & 394.76 \\
$R_{55}$ & 454.06 & 380.44 & 540.47 & $S_{55}$ & 3140.3 & 3415.2 & 2852.1 \\
$R_{66}$ & 3100.7 & 2570.9 & 3734.5 & $S_{66}$ & 25770 & 27917 & 23491 \\
$R_{01}$ & 0.7854 & 0.7413 & 0.8291 & $S_{01}$ & 1.9704 & 2.2033 & 1.7362 \\
$R_{02}$ & 1.0965 & 1.0189 & 1.1756 & $S_{02}$ & 3.4278 & 3.8452 & 3.0106 \\
$R_{03}$ & 1.6781 & 1.5379 & 1.8235 & $S_{03}$ & 6.4554 & 7.2607 & 5.6541 \\
$R_{04}$ & 2.7808 & 2.5187 & 3.0582 & $S_{04}$ & 13.013 & 14.670 & 11.371 \\
$R_{05}$ & 4.9452 & 4.4347 & 5.4956 & $S_{05}$ & 27.846 & 31.452 & 24.283 \\
$R_{06}$ & 9.3705 & 8.3331 & 10.507 & $S_{06}$ & 62.835 & 71.090 & 54.696 \\
$R_{12}$ & 2.0708 & 1.8801 & 2.2700 & $S_{12}$ & 6.7216 & 7.4843 & 5.9546 \\
$R_{13}$ & 3.6033 & 3.2241 & 4.0077 & $S_{13}$ & 13.736 & 15.281 & 12.183 \\
$R_{14}$ & 6.6233 & 5.8544 & 7.4594 & $S_{14}$ & 29.720 & 33.043 & 26.380 \\
$R_{15}$ & 12.846 & 11.239 & 14.624 & $S_{15}$ & 67.690 & 75.224 & 60.106 \\
$R_{16}$ & 26.226 & 22.751 & 30.130 & $S_{16}$ & 161.49 & 179.42 & 143.43 \\
$R_{23}$ & 7.4624 & 6.5642 & 8.4413 & $S_{23}$ & 30.556 & 33.764 & 27.301 \\
$R_{24}$ & 14.932 & 12.983 & 17.094 & $S_{24}$ & 70.220 & 77.429 & 62.879 \\
$R_{25}$ & 31.172 & 26.845 & 36.048 & $S_{25}$ & 168.72 & 185.76 & 151.32 \\
$R_{26}$ & 67.907 & 58.022 & 79.204 & $S_{26}$ & 422.43 & 464.55 & 379.28 \\
$R_{34}$ & 33.644 & 28.891 & 39.007 & $S_{34}$ & 172.02 & 188.70 & 154.89 \\
$R_{35}$ & 74.935 & 63.791 & 87.690 & $S_{35}$ & 433.40 & 474.41 & 391.06 \\
$R_{36}$ & 173.03 & 146.26 & 204.06 & $S_{36}$ & 1132.8 & 1238.1 & 1023.7 \\
$R_{45}$ & 182.43 & 153.93 & 215.50 & $S_{45}$ & 1148.7 & 1252.6 & 1040.5 \\
$R_{46}$ & 444.11 & 372.37 & 528.28 & $S_{46}$ & 3122.2 & 3398.4 & 2833.0 \\
$R_{56}$ & 1160.3 & 966.78 & 1389.7 & $S_{56}$ & 8847.7 & 9603.7 & 8050.0 \\
\hline & & & & & & & \\
\hline
\end{tabular}

Table 1. Values of the coefficients defined by equations (13) and (14), as a function of the ratio $\mu$ between the mass $m_{b}$ of a solvent atom and the mass $m_{a}$ of a solute atom. 


\begin{tabular}{|c|c|c|c|c|c|}
\hline$J$ & $W(1,0)$ & $y_{m}$ & $\rho_{7 M}$ & $10^{6}\left(A / \Phi_{0}\right)^{2}$ & \\
\hline 0 & 1.5957 & 0.04532 & 0.5620 & 0.593 & \\
\hline 1 & 1.8304 & 0.04692 & 0.4470 & 0.541 & $f_{1}=0.3637$ \\
\hline 2 & 1.8945 & 0.04731 & 0.4173 & 0.523 & $f_{1}=0.3608, f_{2}=0.008925$ \\
\hline 3 & 1.8988 & 0.04734 & 0.4161 & 0.522 & $\begin{array}{l}f_{1}=-0.2094, f_{2}=0.3761 \\
f_{3}=-0.08119\end{array}$ \\
\hline 4 & 1.8989 & 0.04734 & 0.4161 & 0.522 & $\begin{array}{l}f_{1}=-0.1386, f_{2}=0.2937 \\
f_{3}=-0.04038, f_{4}=-0.006778\end{array}$ \\
\hline 5 & 1.8986 & 0.04734 & 0.4163 & 0.523 & $\begin{array}{l}f_{1}=0.3868, f_{2}=-0.6868 \\
f_{3}=0.7488, f_{4}=-0.2808 \\
f_{5}=0.03390\end{array}$ \\
\hline 6 & 1.8983 & 0.04734 & 0.4165 & 0.523 & $\begin{array}{l}f_{1}=0.1290, f_{2}=-0.08549 \\
f_{3}=0.1633, f_{4}=-0.01679 \\
f_{5}=-0.02016, f_{6}=0.003959\end{array}$ \\
\hline
\end{tabular}

Table 2. Progressive approach to the solution of the integral equation (5). For each value of $J$, the order of the polynomial appearing in equation (9), the last column provides the coefficients $f_{j}=\alpha_{j} / \alpha_{0}$. The whole table refers to ${ }^{7} \mathrm{Li}$ at the melting point. 


$\begin{array}{ccccc}J & D^{6 / 6} / D^{7 / 7} & n & D^{6 / 7} & D^{7 / 6} \\ ---------- \\ 0 & 1.256 & 2.749 & 6.37 & 6.37 \\ 1 & 1.250 & 2.679 & 6.39 & 6.31 \\ 2 & 1.244 & 2.666 & 6.38 & 6.29 \\ 3 & 1.244 & 2.666 & 6.38 & 6.29 \\ 4 & 1.244 & 2.666 & 6.38 & 6.29 \\ 5 & 1.244 & 2.666 & 6.38 & 6.29 \\ 6 & 1.244 & 2.667 & 6.38 & 6.29\end{array}$

Table 3. Dependence of the isotope effects on the order of the polynomial representing the trial function. The results appearing in the $2^{\text {nd }}, 4^{\text {th }}$ and $5^{\text {th }}$ column refer to lithium at the melting point, while the $3^{\text {rd }}$ column provides the exponent of the power law which is assumed to describe the temperature dependence of $D^{6 / 6}$. Both $D^{6 / 7}$ and $D^{7 / 6}$ are given in $10^{-9} \mathrm{~m}^{2} / \mathrm{s}$. 


\section{FIGURE CAPTIONS.}

Figure 1. Gain in linearity of function $L_{J}(u)$ (see text), as produced by increasing from $J=0$ to $J=6$ the order of the polynomial by which the solution of the integral equation (9) is approximated.

Figure 2. Convergence toward the true solution of the integral equation (5). The three curves correspond to polynomials of order 4, 5 and 6 , by which the above solution is approximated.

Figure 3. Comparison between theoretical and experimental diffusivities for a mixture of liquid lithium isotopes at the melting point. The straight lines denoted by $a$ and $b$ represent the theoretical predictions of the diffusion coefficients of ${ }^{6} \mathrm{Li}$ and of ${ }^{7} \mathrm{Li}$, respectively, as a function of the concentration of ${ }^{7} \mathrm{Li}$ in the mixture. The corresponding experimental points are taken from Feinauer et al. [3,4]. 


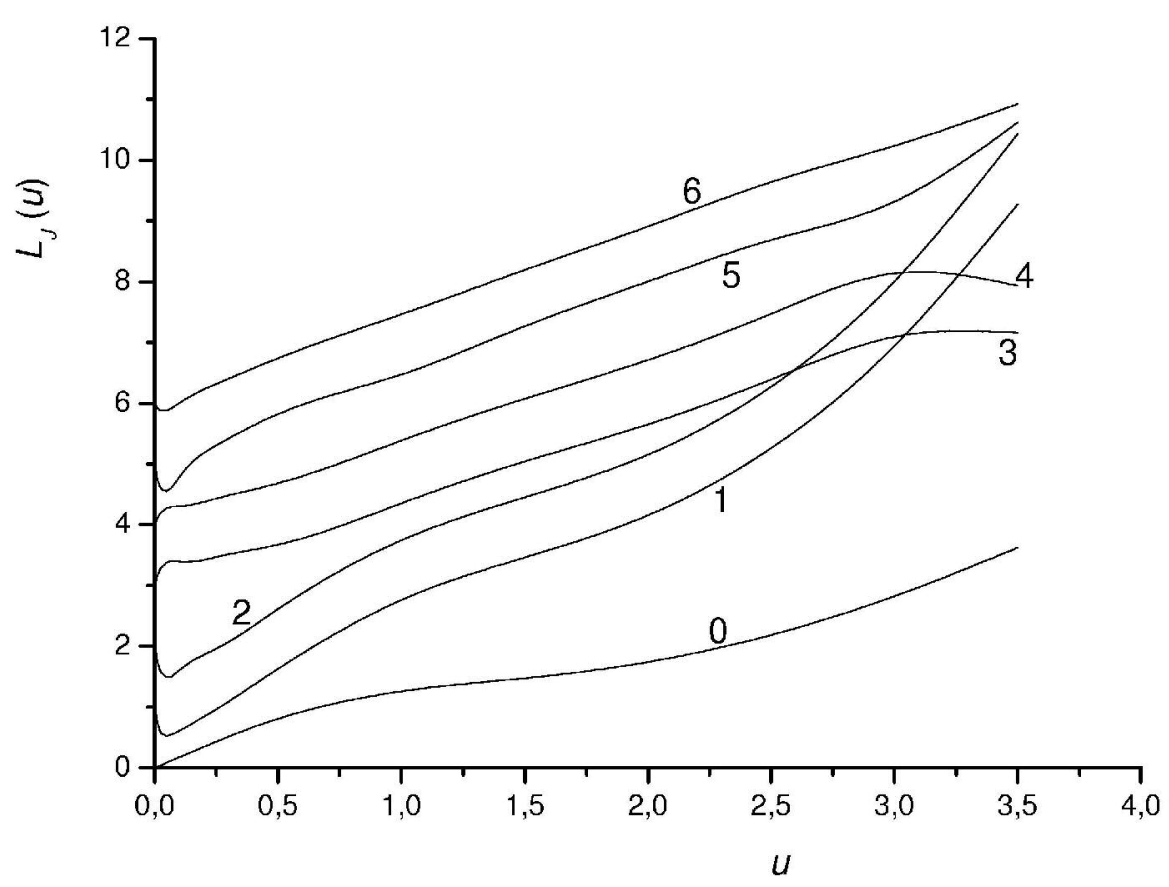

Figure 1

$103 \times 80 \mathrm{~mm}(600 \times 600 \mathrm{DPI})$ 


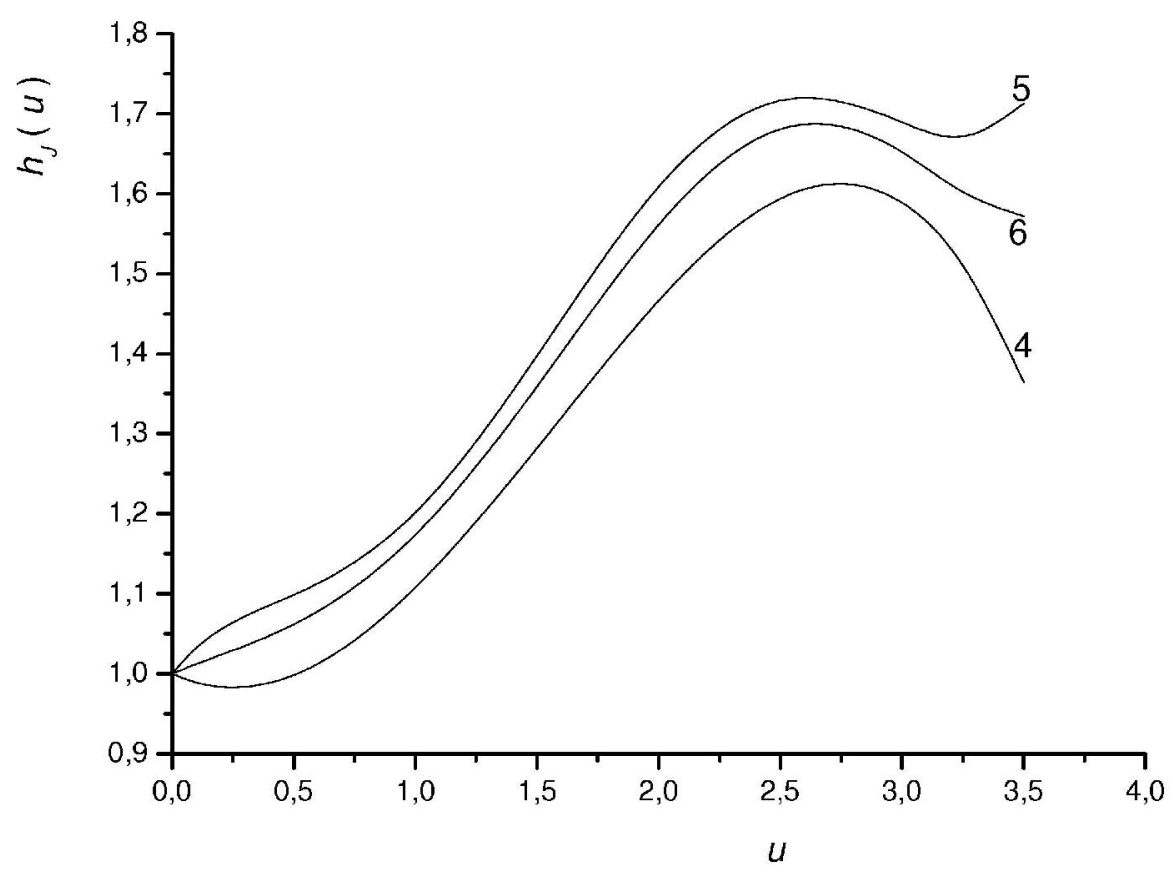

Figure 2

$104 \times 80 \mathrm{~mm}(600 \times 600$ DPI $)$ 


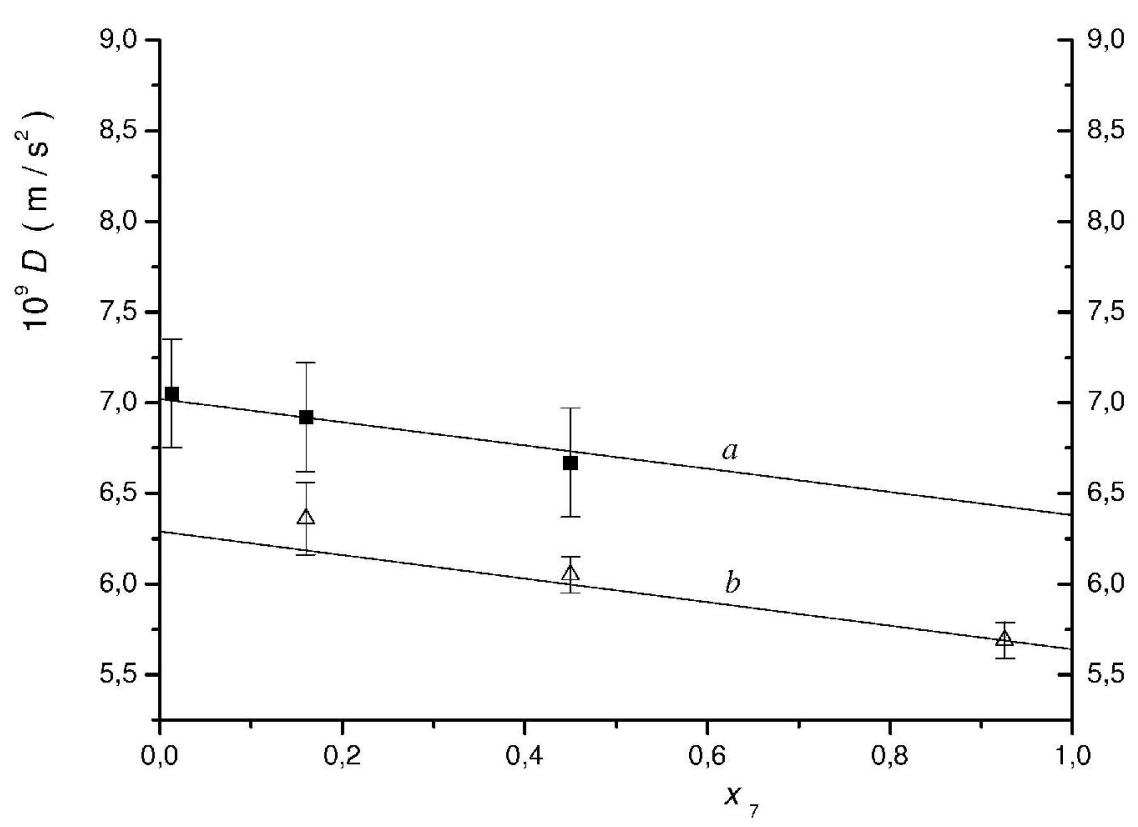

Figure 3

$110 \times 80 \mathrm{~mm}(600 \times 600 \mathrm{DPI})$ 